\title{
All-trans retinoic acid inhibits proliferation, migration, invasion and induces differentiation of hepa1-6 cells through reversing EMT in vitro
}

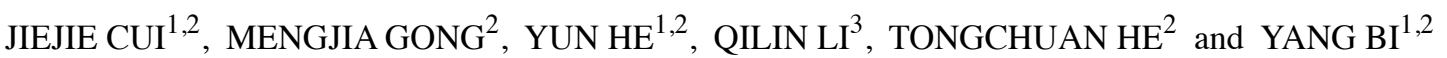 \\ ${ }^{1}$ Department of Pediatric Surgery, ${ }^{2}$ Stem Cell Biology and Therapy Laboratory, Ministry of Education \\ Key Laboratory of Child Development and Disorders, Chongqing Stem Cell Therapy Engineering Technical Center, \\ The Children's Hospital of Chongqing Medical University; ${ }^{3}$ Department of Ultrasound, \\ The Third People's Hospital of Chongqing, Chongqing 400014, P.R. China
}

Received August 19, 2015; Accepted October 20, 2015

DOI: 10.3892/ijo.2015.3235

\begin{abstract}
Hepatocellular carcinoma (HCC) has the characristics of tumor invasiveness, frequent intrahepatic spread and extra hepatic metastases, which affects the therapy efficiency and prognosis. Epithelial-mesenchymal transition (EMT) is now recognized as a key process in tumor invasion, metastasis and the generation of cancer initiating cells. All-trans retinoic acid (ATRA) is currently used as a potential chemo-therapeutic or chemo-preventive agent because of its anti-proliferative, pro-apoptotic and antioxidant properties. This study investigated the effects of ATRA at different concentrations on the proliferation, migration, invasion, differentiation and functions of the mouse hepa1-6 hepatocarcinoma cell line and explored whether ATRA regulates EMT in the antitumor process. Trypan blue staining and colony formation assay were used to detect cell proliferation. Wound-healing assay and Transwell Matrigel assay were performed to examine migration. Invasion was assessed by using Transwell invasion assay. In the present study, ATRA significantly inhibited the cell growth, colony formation, migration, and invasion capability of hepa1-6 cells in a dose-dependent manner. Furthermore, ATRA at low concentration $(0.1 \mu \mathrm{mol} / \mathrm{l})$ could generate these
\end{abstract}

Correspondence to: $\mathrm{Dr}$ Yang $\mathrm{Bi}$, Department of Pediatric Surgery, Stem Cell Biology and Therapy Laboratory, The Children's Hospital of Chongqing Medical University, Building 7, Room 905, 136 Zhongshan Er Road, Yuzhong, Chongqing 400014, P.R. China E-mail: yangbi1981@cqmu.edu.cn

Abbreviations: ATRA, all-trans retinoic acid; HCC, hepatocellular carcinoma; EMT, epithelial-mesenchymal transition; ICG, indocyanine green; PAS, periodicacid-schiff; ALB, albumin; AFP, $\alpha$ fetoprotein; CK18, cytokeratin 18; TAT, tyrosine aminotransferase; ApoB, apolipoprotein B

Key words: hepatocarcinoma cell, all-trans retinoic acid, epithelialmesenchymal transition, proliferation, migration, invasion, differentiation influences. After treated in the ATRA medium, the expression of mature hepatic markers ALB (albumin), CK18 (cytokeratin 18), TAT (tyrosine aminotransferase), ApoB (apolipoprotein B) decreased and that of hepatocarcinoma marker AFP ( $\alpha$ fetoprotein) increased. At day 7 after ATRA induction, hepa1-6 cells showed comparable indocyanine green (ICG) uptake and glycogen storage function to the blank control. The mRNA expression of mesenchymal markers N-cadherin, vimentin, snail and twist decreased, while expression of epithelial marker E-cadherin increased in hepa1-6 cells after treated with ATRA. Therefore, this study demonstrates that ATRA remarkably suppressed the proliferation, migration, invasion of hepa1-6 hepatocarcinoma cell line and effectively induced its differentiation and liver functions in vitro through the reversal of EMT. HCC may be more sensitive to ATRA than other cancers, suggesting the prospective usefulness of ATRA in the treatment of HCC.

\section{Introduction}

Hepatocellular carcinoma (HCC) is the sixth most common cancer and the third most frequent cause of cancer death worldwide (1). Although partial liver resection and liver transplantation have significantly improved survival in patients with small tumors, the prognosis for HCC remains poor because of tumor invasiveness, frequent intrahepatic spread and extrahepatic metastases (2). Thus, effective non-surgical strategies and identification of the molecular mechanisms are crucially responsible for control of the growth, invasive and metastatic potential of HCC.

Therefore, a clearer understanding of the molecular mechanisms underlying tumor invasiveness and migration is essential for the development of new therapies for HCC. Epithelial-mesenchymal transition (EMT) is defined as a process during which epithelial cells lose their cell polarity, phenotypic characteristics and acquire mesenchymal cell features (3). It has been suggested that EMT might be closely associated with the acquisition of aggressive traits by tumor cells, thus facilitating the early stages of metastasis and the subsequent dissemination of carcinoma cells (4-6). Increasing 
evidence indicates that aberrant regulation of EMT is a determinant of the cancer cell invasive and metastatic behavior. Therefore, targeting EMT may serve as an efficient strategy for the treatment of malignant and metastatic tumors.

All-trans retinoic acid (ATRA), as a predominant natural metabolite of vitamin $\mathrm{A}$, is involved in many important biological processes, including vision, morphogenesis, differentiation, growth, metabolism, and cellular homeostasis (7). ATRA is currently used to induce remission in patients with acute promyelocytic leukemia and has the potential for use in the treatment of solid tumors including HCC (8-11). Many studies have reported that ATRA has anti-HCC ability. Additionally, ATRA combined with other chemotherapeutic agents could induce cancer cell differentiation, increase the sensitivity of hepatocarcinomas to chemotherapy, reduce cell migration in vitro and metastasis in vivo $(12,13)$. However, the mechanisms underlying the effect of ATRA on HCC are largely unknown. It has been revealed that ATRA can induce mesenchymal to epithelial transition of HCT116 cells (10), while ATRA modulates epithelial-to-mesenchymal-transition of mammary tumor cells via the TGF $\beta$ and NOTCH pathways (11). Thus, we raised a hypothesis that the antiHCC effect of ATRA might be closely related to the reverse process of EMT.

In this study, we demonstrated that ATRA could inhibit the proliferation, migration, invasion of mouse hepa1-6 hepatocarcinoma cell lines, as well as induce their differentiation and hepatic function. Then, we found that the antitumor process was correlated with the decreased expression of mesenchymal marker genes and the increased expression of epithelial marker genes by regulation of ATRA. Our study may support the understanding of anti-HCC effect of ATRA and promote its clinical application to this disease.

\section{Materials and methods}

Cell and chemicals. The mouse hepa1-6 hepatocarcinoma cell lines was purchased from the American Type Culture Collection (Manassas, VA, USA) and maintained in complete Dulbecco's modified Eagle's medium (DMEM, Gibco Life Technologies, Carlsbad, CA, USA) supplemented with $10 \%$ fetal bovine serum (FBS, Gibco Life Technologies), $100 \mathrm{U} / \mathrm{ml}$ penicillin, and $100 \mu \mathrm{g} / \mathrm{ml}$ streptomycin at $37^{\circ} \mathrm{C}$ in $5 \% \mathrm{CO}_{2}$. Unless indicated otherwise, all chemicals were purchased from Sigma-Aldrich (St. Louis, MO, USA), all antibodies were purchased from Santa Cruz (USA). PCR primers were synthesized by Huada Gene Inc (Shenzhen, China).

Treatment of ATRA. The Hepa1-6 cells were treated with complete DMEM medium containing different concentrations of ATRA $(0.1,1.0$ and $10.0 \mu \mathrm{mol} / \mathrm{l})$. The culture was replaced every three days. The group without ATRA treatment was set up as control group. Three independent experiments were performed in duplicate.

Trypan blue staining. Cells were incubated in 24-well plates at $5.0 \times 10^{4}$ cells per well with different concentrations of ATRA treatment. Cell viability was measured by trypan blue staining after treatment at days 0,2,4 and 6. Both adherent and suspended cells were collected and mixed with $0.4 \%$
2X trypan blue buffer. Cell mixture $(10 \mu \mathrm{l})\left(\sim 10^{6}\right.$ cells $\left./ \mathrm{ml}\right)$ was counted using hemocytometer under a microscope (Nikon Eclipse Ti, Japan). The dead cells were stained blue. The mean and standard deviation were calculated.

Colony formation assay. The colony formation rate indicates the independent proliferation and viability of cells. Briefly, logarithmic phase cells were planted in 12-well plates at 500 cells per well. Culture medium was replaced when the generative cells attached to the wall. After incubation for 20 days, the visible colonies were fixed with $4 \%$ paraformaldehyde for $30 \mathrm{~min}$ and then stained with $0.1 \%$ crystal violet for 10 min. Cells were washed twice with PBS after each step. The cell colonies were observed and counted under the inverted microscope. Fifty cells are equivalent to one colony and the cell colony formation rate was calculated as follows, $\mathrm{R}_{\mathrm{cf}}=$ $\mathrm{N}_{\mathrm{cf}} / \mathrm{Nvc} \times 100 \%$, where $\mathrm{R}_{\mathrm{cf}}$ is the rate of colony formation, $\mathrm{N}_{\mathrm{cf}}$ is the number of colonies formed, $\mathrm{N}_{\mathrm{vc}}$ is the number of vaccinated cells.

Wound-healing assay. To determine the cell migratory ability, the classical scratch would-healing assay was performed as described (14). Briefly, 5.0x $10^{4}$ cells per well were incubated in 6 -well plates. A linear wound was made by scraping a pipette tip across the cell layer. After washing three times with PBS to remove floating cells and debris, cells were incubated in culture medium with different concentrations of ATRA. Then the scratched fields were photographed at 0,24 and $48 \mathrm{~h}$ to calculate the wound healing.

Transwell migration and invasion assay. For Transwell invasion assay, logarithmic phase cells were trypsinized and re-suspended in DMEM/ATRA without FBS. Cell suspension (200 $\mu \mathrm{l}$ ) was added to the Transwell upper chamber coated with Matrigel membrane in triplicate. DMEM culture solution containing $10 \%$ FBS was added to the lower chamber of each well and incubated for $48 \mathrm{~h}$ at $37^{\circ} \mathrm{C}$. The non-invasive cells on the upper surface of the membrane were removed and the invasive cells were stained in $100 \mu$ crystal violet (Bioteke, Beijing, China). The invasive cells in five random high power fields were counted. The Transwell migration assay was the same as the invasion assay, with the exception of the Matrigel coating. The cells were stained and counted, as aforementioned.

RNA extraction and quantitative real-time polymerase chain reaction (real-time $q P C R$ ). Cells were incubated with different ATRA treatments for 2, 4 and 7 days. As previously described (15), total RNA was extracted by using an RNA Extraction kit (Bioteke) according to the manufacturer's instructions and then reverse transcribed into cDNA by using Superscript II reverse transcriptase (Thermo Fisher Scientific, USA). The PCR templates were prepared with 5- to 10-fold dilution of the first strand cDNA products. The cDNA samples at days 2 and 4 were used to detect the EMT genes (E-cadherin, $\mathrm{N}$-cadherin, vimentin, snail, twist and fibronectin). $\mathrm{N}$-cadherin, vimentin, snail, twist and fibronectin are mesenchymal markers, E-cadherin is an epithelial marker $(16,17)$. The cDNA samples at day 7 were detected for the differentiation genes as follows ALB (albumin), CK18 (cytokeratin 18), TAT 
Table I. Sequences of primers for real-time qPCR.

Sequences of primers (5'-3')

\begin{tabular}{lll}
\cline { 2 - 3 } Name of genes & \multicolumn{1}{c}{ Forward } & \multicolumn{1}{c}{ Reverse } \\
\hline$\beta$-actin & AGGGAAATCGTGCGTGAC & CGCTCGTTGCCAATAGTGA \\
ALB & CCAGACATTCCCCAATGC & CAAGTTCCGCCCTGTCAT \\
AFP & ACGAGGAAAGCCCCTCAG & GCCATTCCCTCACCACAG \\
CK18 & CTGGGCTCTGTGCGAACT & ACAGAGCCACCCCAGACA \\
TAT & ACCTTCAATCCCATCCGA & TCCCGACTGGATAGGTAG \\
ApoB & CATGTGATCCCCACAGCA & TCCCAGGACCATGGAAAA \\
E-cadherin & CAAGGACAGCCTTCTTTTCG & TGGACTTCAGCGTCACTTTG \\
N-cadherin & CTGGGACGTATGTGATGACG & TGATGATGTCCCCAGTCTCA \\
vimentin & CAGATGCGTGAGATGGAAGA & TCCAGCAGCTTCCTGTAGGT \\
snail & AAACCCACTCGGATGTGAAG & GAAGGAGTCCTGGCAGTGAG \\
twist & CAGCGGGTCATGGCTAACG & CTTGTCCGAGGGCAGCGT \\
fibronectin & GCAGAACCAGAGGAGGCACA & CAATGGCGTAATGGGAAACC
\end{tabular}

(tyrosine aminotransferase), ApoB (apolipoprotein B), and AFP ( $\alpha$ fetoprotein). The genes of interest were amplified with qPCR primers which were designed using the Primer 3.0 program (Table I). A real-time PCR protocol for real-time PCR amplification was performed as follows: $72^{\circ} \mathrm{C} \times 3 \mathrm{~min}$, $94^{\circ} \mathrm{C} \times 3 \mathrm{~min}, 92^{\circ} \mathrm{C}$ × $20 \mathrm{sec}, 72^{\circ} \mathrm{C}$ × $20 \mathrm{sec}, 9$ cycles, with $1^{\circ} \mathrm{C}$ degree decrease per cycle, followed by $94^{\circ} \mathrm{C} \times 20 \mathrm{sec}$, $55^{\circ} \mathrm{C}$ × $20 \mathrm{sec}, 72^{\circ} \mathrm{C}$ x $20 \mathrm{sec}$ for $25-30$ cycles and $72^{\circ} \mathrm{C} \times 3 \mathrm{~min}$. All samples were normalized by endogenous levels of $\beta$-actin.

Western blot analysis. After treatment of ATRA for 7 days, total proteins of each group were extracted. Thereafter $20 \mu \mathrm{g}$ of total protein per group were separated on a $10 \%$ SDS-PAGE (Beyotime Institute of Biotechnology, Shanghai, China) and electrophoretically transferred to PVDF membrane (Milipore, Billerica, MA, USA). After blocked with $5 \%$ fat-free skim milk at room temperature for $2 \mathrm{~h}$, membranes were incubated at $4^{\circ} \mathrm{C}$ overnight with primary antibodies against AFP (1:200; goat. no. sc-8108; Santa Cruz Biotechnology, Inc., Dallas, TX, USA), ALB (1:200; goat. no. sc-46293; Santa Cruz Biotechnology, Inc.), CK18 (1:200; mouse. no. sc-51582; Santa Cruz Biotechnology, Inc.) and $\beta$-actin (1:200; mouse. no. sc-47718; Santa Cruz Biotechnology, Inc.), followed by probing with the appropriate second antibody at room temperature for $1 \mathrm{~h}$. The presence of the proteins of interest was detected by using the G-BOX iChemi XR gel documentation system (Syngene, Cambridge, UK).

Immunofluorescence staining. Cells receiving the indicated treatments were cultured in 24-well plates for 7 days; immunofluorescence staining was carried out as previously reported (15). Cells were fixed with $4 \%$ paraformaldehyde for $15 \mathrm{~min}$ and then perforated with $5 \%$ triton for $20 \mathrm{~min}$. The fixed cells were blocked with $5 \%$ goat serum for $1 \mathrm{~h}$, followed by incubation with primary antibodies against AFP and CK18 at $4^{\circ} \mathrm{C}$ overnight, then incubated with DyLight ${ }^{\circledR}$ 594- or 488-conjugated secondary antibody (Jackson ImmunoResearch Laboratories,
Inc., West Grove, PA, USA) for $1 \mathrm{~h}$. Nuclei were stained with DAPI. Cells were washed twice with PBS after each step. The presence of proteins was examined under a fluorescence microscope (TE2000-S, Nikon).

Indocyanine green (ICG) uptake and release. Cells in 24-well plates were washed twice with PBS and incubated with DMEM supplemented with freshly prepared ICG at a final concentration of $1 \mathrm{mg} / \mathrm{ml}$ for $1 \mathrm{~h}$ at $37^{\circ} \mathrm{C} 5 \% \mathrm{CO}_{2}$. Then DMEM was removed and cells were gently washed three times with PBS, the green-stained cells were photographed under a microscope. Complete medium was then added and the cells were incubated for $>6 \mathrm{~h}$; the cells were then observed under a microscope to ensure ICG release. At least 10 non-overlapping fields of vision were recorded.

Periodic acid-Schiff (PAS) staining. Cells cultured as described above were fixed with $4 \%$ paraformaldehyde at day 7. Subsequently, cells were stained by $0.5 \%$ periodic acid for $5 \mathrm{~min}$, and Schiff's solution for $15 \mathrm{~min}$, in turn. All steps were carried out at room temperature and cells were washed with $\mathrm{ddH}_{2} \mathrm{O}$ gently after each step. The positive cells stained purple. More than 10 non-overlapping fields of vision in each group were recorded under a microscope.

Statistical analysis. The data are presented as mean \pm standard deviation (SD) and analyzed using the SPSS 15.0 software package. A two-tailed Student's t-test assuming equal variances was performed to measure significant differences between two groups and variance analysis was performed to measure significant differences among at least three groups. A $\mathrm{p}<0.05$ was considered to be statistically significant.

\section{Results}

ATRA inhibits proliferation and colony formation of hepal-6 cells. As shown in Fig. 1A, ATRA effectively inhibited cell 


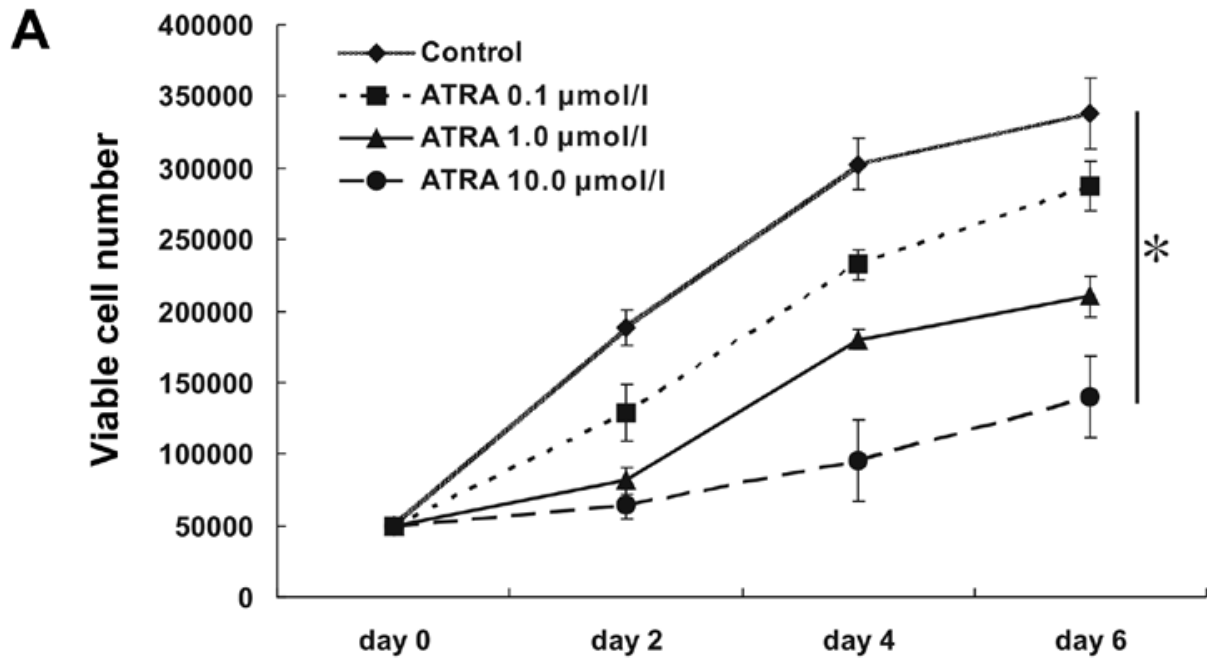

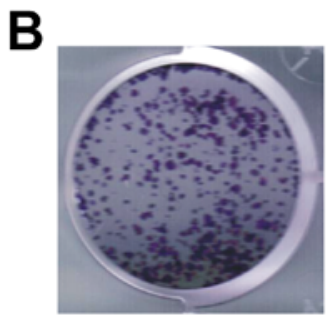

Control

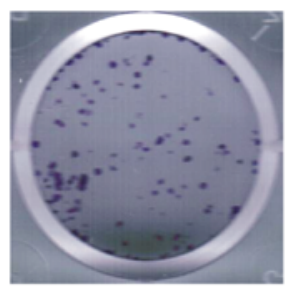

ATRA $1.0 \mu \mathrm{mol} / \mathrm{I}$

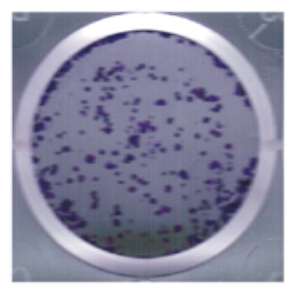

ATRA $0.1 \mu \mathrm{mol} / \mathrm{l}$

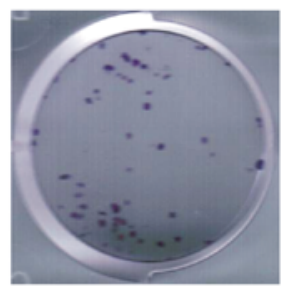

ATRA $10.0 \mu \mathrm{mol} / \mathrm{I}$

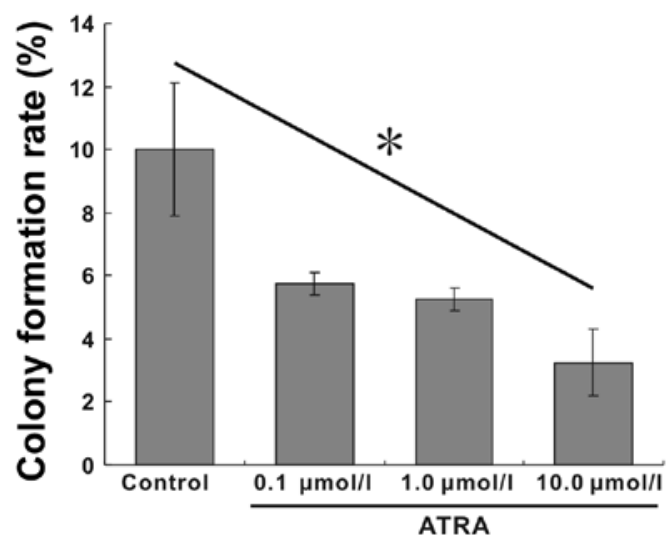

Figure 1. ATRA inhibited hepa1-6 cell proliferation and colony formation in a dose-dependent manner. (A) Cell growth curve was evaluated by trypan blue staining to measure viable cells. (B) Colony formation assay. Three independent experiments were performed in duplicate, and representative results are shown $(\mathrm{p}<0.05)$.

proliferation compared with blank control. The inhibition efficiency of ATRA was enhanced gradually with the increase of concentration, which was strongest at day 6 with $10.0 \mu \mathrm{mol} / 1$ ATRA treatment. The colony formation rate indicates the independent viability of cells. Treatment with ATRA significantly exhibited inhibitory effects on cell colony formation. Moreover, the rate of cell colony formation also decreased gradually with the increase of ATRA concentration. The colony formation rates of ATRA at concentration of 0, 0.1, $1.0,10.0 \mu \mathrm{mol} / 1$ were $10,5.75,5.25$ and $3.25 \%$, respectively (Fig. 1B, p<0.05). Therefore, this result suggested that ATRA inhibited cell proliferation in a dose-dependent manner.

ATRA suppresses the migration and invasion of hepal-6 cells. HCC has highly metastatic capacity because of its strong migratory ability. Wound-healing assay was used to detect the horizontal migration capability of cells. We found that ATRA inhibited the horizontal migration ability of hepa1- 6 cells (Fig. 2A). The similar results of Transwell migration assay showed, that cell migration followed a downward trend as the concentration of ATRA increased. The migration rates in $0,0.1$, 1.0, $10.0 \mu \mathrm{mol} / \mathrm{l}$ ATRA treated groups were 52.14, 45.17, 29.24 and $22.68 \%$, respectively, the migration rate of $10.0 \mu \mathrm{mol} / 1$ group declined by $>56.50 \%$ compared with blank control (Fig. 2B, p<0.05). The invasive ability of the cells induced by ATRA was detected by the Matrigel invasion assay. The invasive ability of hepa1- 6 cells after ATRA treatment was remarkably attenuated and also exhibited a dose-dependent manner. The positive invasive rates at corresponding ATRA concentration were $6.25,3.92,3.36$ and $3.12 \%$, respectively, the invasive rate of $10.0 \mu \mathrm{mol} / 1$ group reduced by $50 \%$ compared with blank control (Fig. 3, p<0.05). These results indicated that ATRA possessed excellent inhibitory effects on the migration and invasion of hepa1- 6 cells.

ATRA induces the differentiation of hepal-6 cells. Due to the inhibitory ability of ATRA on mesenchymal phenotype in hepa1-6 cells, we tried to further detect the regulation of ATRA on its epithelial phenotype. ALB, CK18, TAT, and ApoB are important molecules associated with mature hepato- 


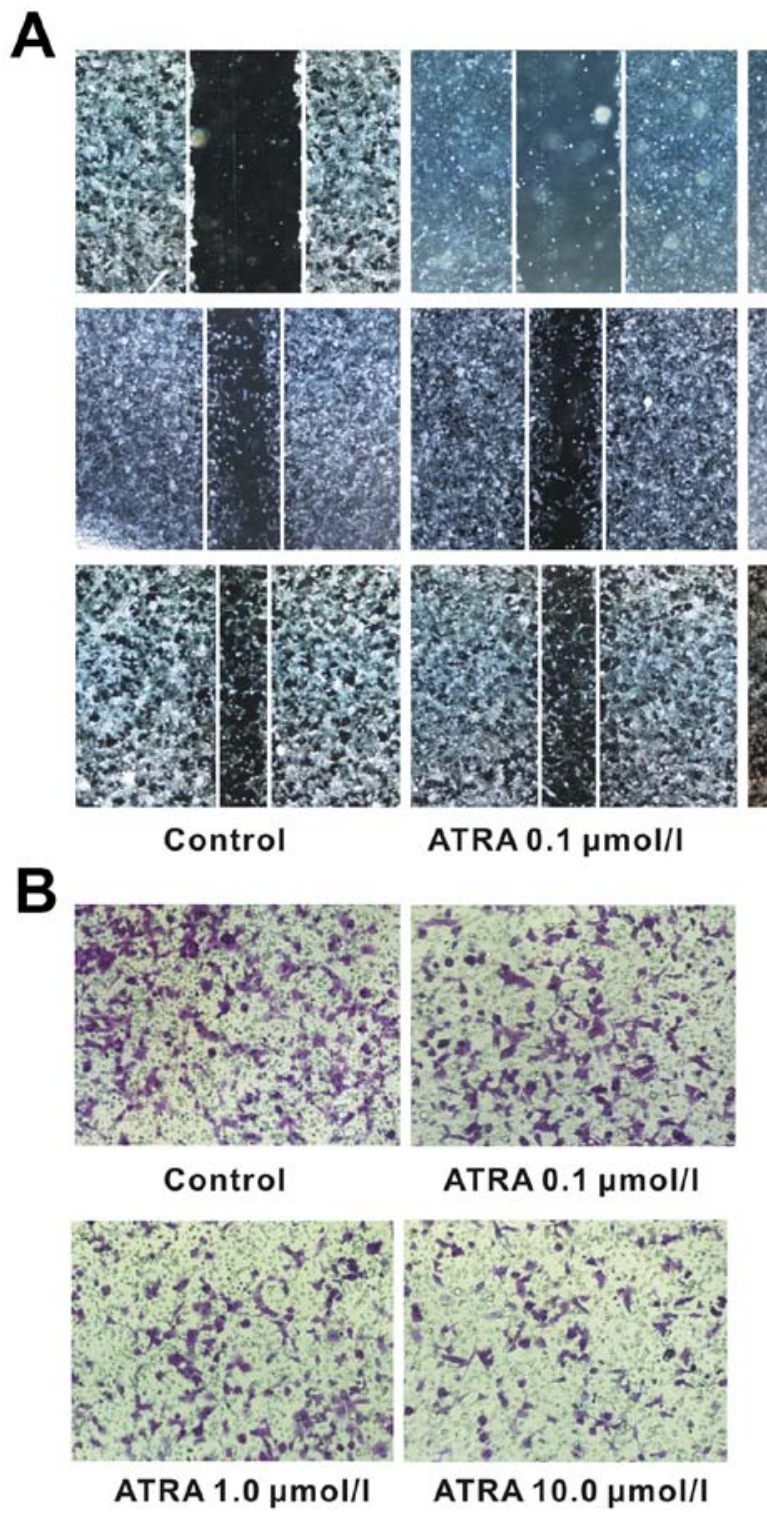

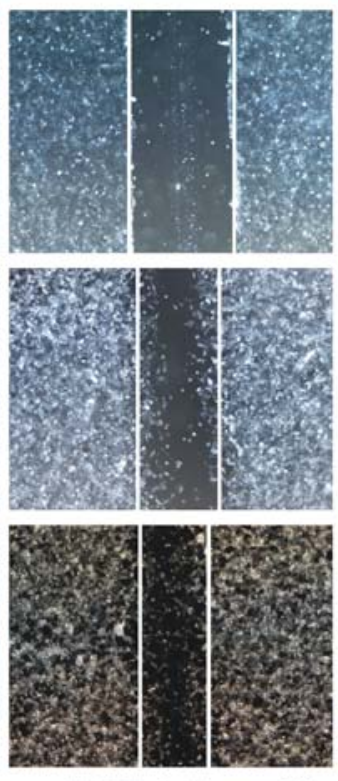

ATRA $1.0 \mu \mathrm{mol} / \mathrm{l}$

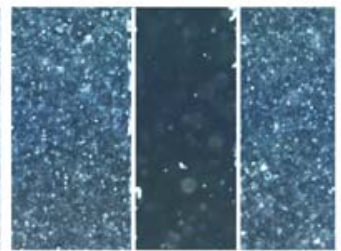

$\mathrm{Oh}$

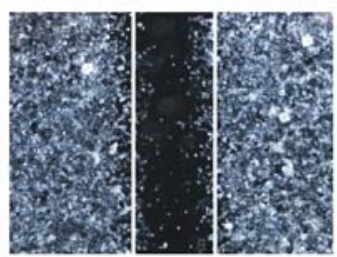

$24 \mathrm{~h}$

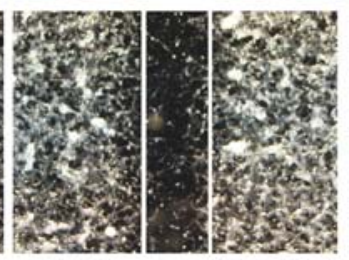

ATRA $10.0 \mu \mathrm{mol} / \mathrm{I}$

\section{$48 \mathrm{~h}$}

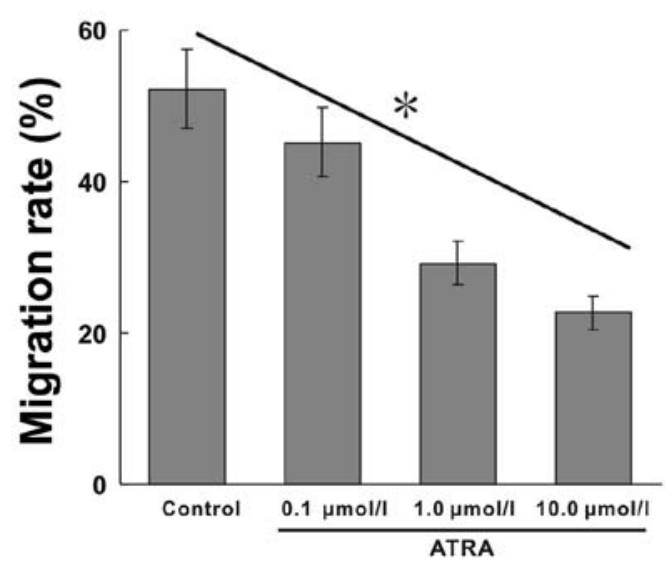

Figure 2. ATRA inhibits the migration of hepa1-6 cells. (A) The migration of hepa1-6 cells were determined by Wound-healing assay. (B) Transwell migration assay. The number of migratory cells detected by Transwell migration assay at different concentrations of ATRA ( $\left.{ }^{*} \mathrm{p}<0.05\right)$.

cytes. As shown in Fig. 4A, all the mRNA expression of these markers were upregulated by ATRA. Moreover, the regulation effect appeared to be strengthened with the increasing concentration of ATRA $(\mathrm{p}<0.05)$. Additionally, AFP, a marker of hepatocarcinoma, was downregulated $(\mathrm{p}<0.05)$. The patterns of the protein expression of ALB, CK18 and AFP as detected by the western blotting results (Fig. 4B), largely corresponded to those observed by quantitative real-time PCR. In addition, immunofluorescence staining carried out to detect the protein expression of AFP and CK18 also showed a similar trend (Fig. 4C). All these results indicated that ATRA could effectively induce hepa1-6 cells to mature hepatocytes at least in the mRNA and protein levels.

ATRA induces hepal-6 cells to display functions. To further substantiate the hypothesis that ATRA may induce hepa1-6 cells to be functional cells; we performed ICG uptake and PAS staining. The evaluation of ICG uptake is a common way to estimate liver function (18). As shown in Fig. 5, almost no the green-positive cells were observed in blank control, while many areas of cells in the treatment groups were stained green. On the other hand, mature hepatocytes have the ability of glycogen synthesis and storage. PAS staining method is used to detect glycogen which is displayed by a purple color in the cytoplasm (19). Following ATRA treatment indicated above, the purple color in cytoplasm was significantly increased. Furthermore, with the increasing concentration of ATRA, the positive rate in both ICG uptake and PAS staining were increasing. Taken together, these results suggested that ATRA induction could not only improve the expression of hepatic markers and inhibit hepatocarcinoma markers, but also effectively induced hepa1-6 cells to display functions similar to those of mature hepatocytes.

ATRA regulates the expression of EMT markers. Finally, we investigated whether the regulation effect of ATRA on 


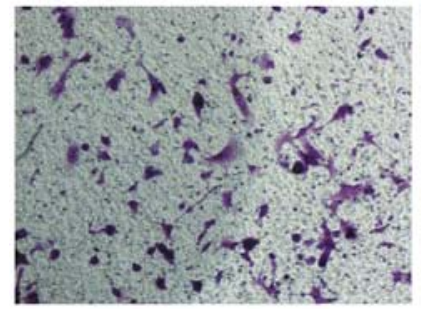

Control

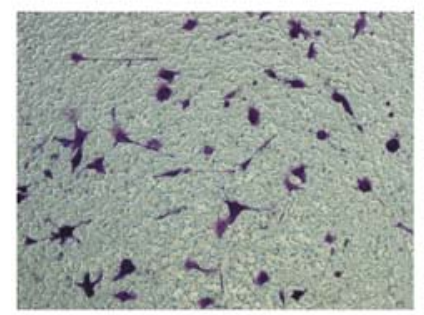

ATRA $1.0 \mu \mathrm{mol} / \mathrm{I}$

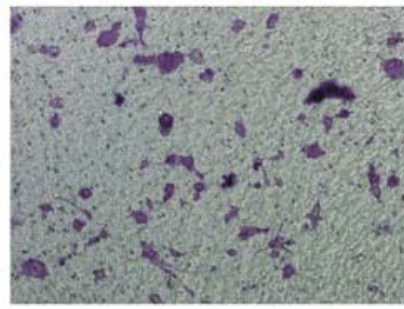

ATRA $0.1 \mu \mathrm{mol} / \mathrm{l}$

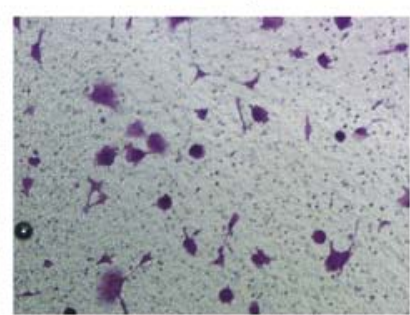

ATRA $10.0 \mu \mathrm{mol} / \mathrm{l}$

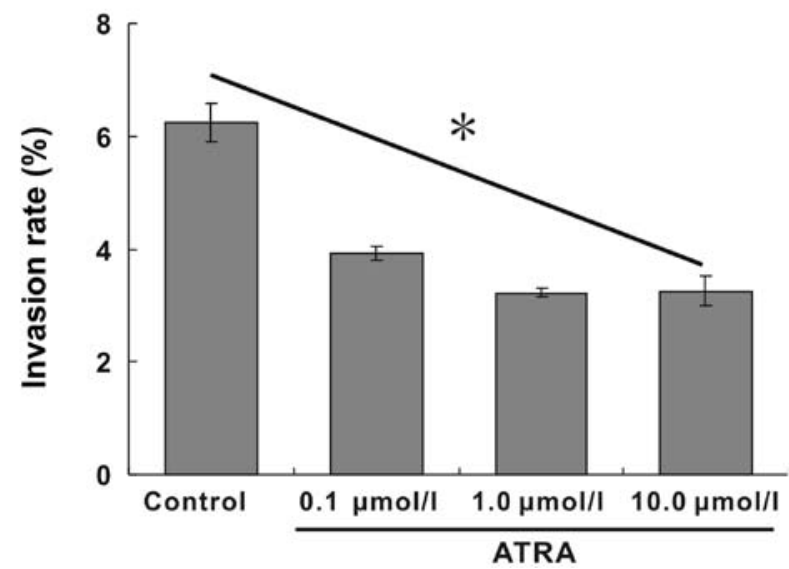

Figure 3. ATRA suppresses the invasion of hepa1-6 cells. Transwell Matrigel invasion assay was used to determine the invasion ability of hepa1- 6 cells receiving treatment of ATRA $\left({ }^{*} \mathrm{p}<0.05\right)$.

hepa1-6 cells had association with EMT. For this purpose, mRNA expression of critical genes for EMT process including E-cadherin, $\mathrm{N}$-cadherin, vimentin, snail, twist, and fibronectin were assessed by real-time PCR at days 2 and 4 . E-cadherin plays a central role in maintaining epithelial cell-cell adhesion and polarity (20). Compared to control, E-cadherin expression had a remarkable increase in the ATRA groups, and its expression between days 2 and 4 had statistical significance. In contrast, ATR A treatment resulted in a strong repression on the expression of mesenchamal markers, including $\mathrm{N}$-cadherin, vimentin, snail and twist. However, there was no difference between days 2 and 4 . Moreover, the expression presented a dose-dependent trend. The expression of fibronectin could not be regulated by ATRA (data not shown). Especially, the expression of E-cadherin of $10.0 \mu \mathrm{mol} / 1$ group at day 4 was remarkably enhanced, with a $>3.7$-fold increase, the expressions of $\mathrm{N}$-cadherin and twist at day 2, vimentin and snail at day 4 in $10.0 \mu \mathrm{mol} / 1$ group were decreased by $75,56.1,51.2$, and 67.7\%, respectively, compared with blank control (Fig. 6, $\mathrm{p}<0.05)$. Therefore, we demonstrated that ATRA was able to reverse EMT process of hepa1- 6 cells by regulating EMT marker genes.

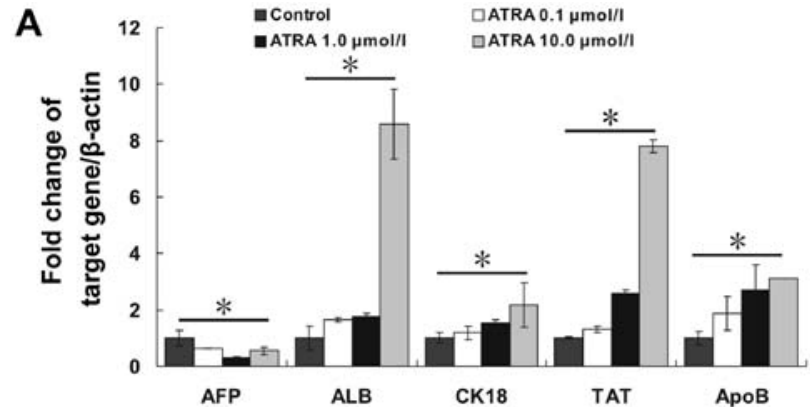

B

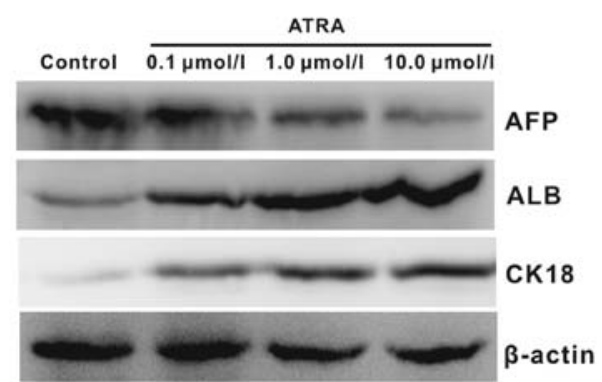

C

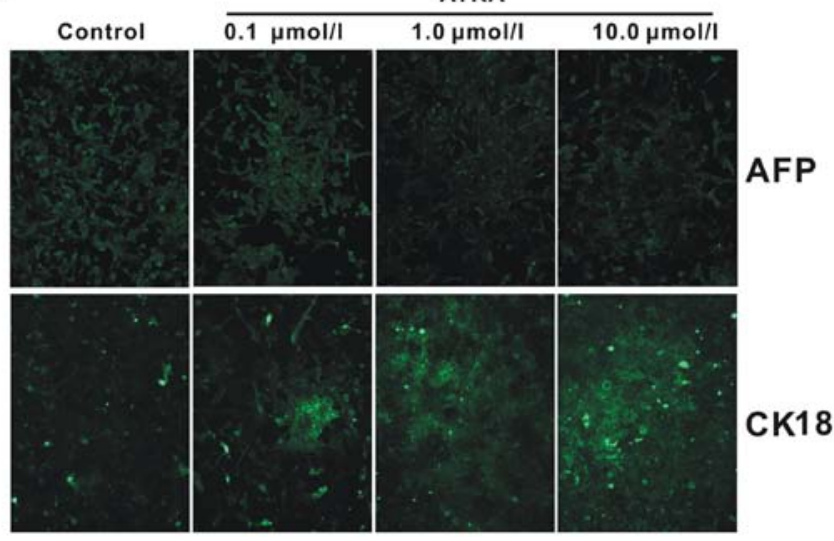

Figure 4. ATRA promotes hepa1-6 cells to functional hepatocytes in the mRNA and protein expression levels. (A) qRT-PCR analysis of the mature hepatocytes and hepatocarcinoma related genes, ALB, CK18, TAT, ApoB and AFP. The qRT-PCR results were confirmed in at least 3 batches of independent experiments, and representative results are shown $\left({ }^{*} \mathrm{p}<0.05\right)$. (B) Western blotting of AFP, ALB and CK18 was analyzed with $\beta$-actin normalization. Each bar represents the mean $\pm \mathrm{SD}, \mathrm{n}=4\left({ }^{*} \mathrm{p}<0.05\right)$. (C) Immunofluorescence staining of AFP and CK18.

\section{Discussion}

Recurrence and metastasis are characteristic features of HCC and the main factors related to poor prognosis in patients with HCC. In recent years, an increasing number of researchers have reported the role for EMT in tumor invasion and metastatic spread. On the other hand, the transition from primary CSCs (cancer stem cells) to EMT-positive CSCs is a significant step in HCC progression. Studies suggest that CSCs having EMT phenotypes are associated with sensitivity and resistance to chemotherapy in various tumor models. Therefore, chemotherapeutics which can inhibit or reverse EMT process would be effective for the treatment of malignant and metastatic tumors including HCC. ATRA, the most important active form of vitamin A, is especially required with respect to embryonic development, growth, 

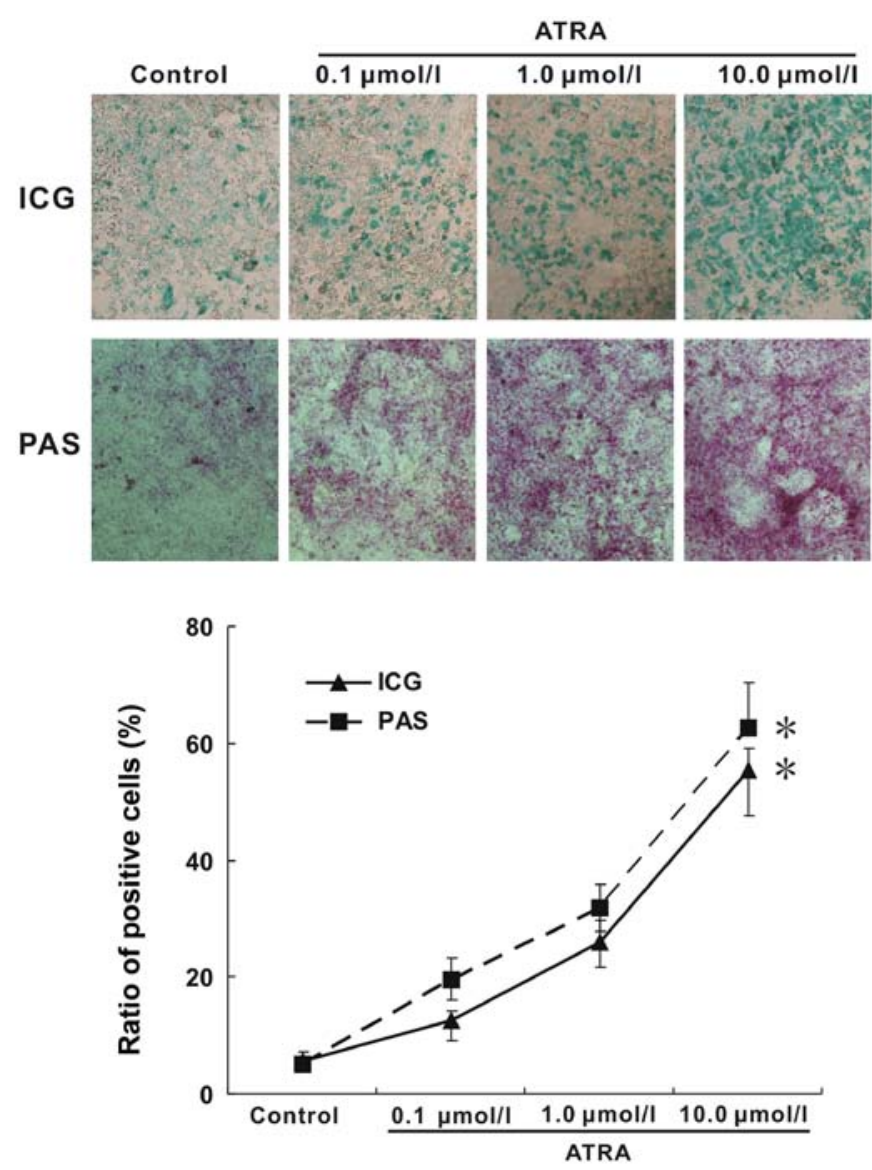

Figure 5. ATRA induces hepa1-6 cells to restore mature hepatocyte functions. Function analysis was performed on day 7 following treatment. Indocyanine green (ICG) uptake assay: cells with a green-stained nucleus are the positive-stained cells. Periodic acid-Schiff (PAS) staining, purple color in cell plasma indicates glycogen accumulation $\left({ }^{*} \mathrm{p}<0.05\right)$.

night vision, and the maintenance of the immune system. ATRA also functions as a hormone-like growth factor for epithelial and other cells, can induce differentiation in many cell types and is the most widely used differentiating therapeutic agent $(21,22)$. ATRA is the first example of a clinically useful cyto-differentiating agent to be used in the treatment of acute promyelocytic leukemia (23). Now it is proposed as an antitumor agent not only in the context of hematological malignancies but also the chemo-prevention and treatment of many cancers including HCC (8-11). In addition, it has been reported that ATRA could be used as a chemopreventive agent to inhibit the progression of premalignant lesions of the breast $(24,25)$. In the present study, we investigated the effect of ATRA on the proliferation, migration, invasion and differentiation of hepatocarcinoma cells and explored whether ATRA regulate EMT in the antitumor process.

In this study, we investigated whether ATRA at different concentrations influence the cell phenotype and biological characteristics of hepatocarcinoma hepa1-6 cells. We found that ATRA significantly inhibited the proliferation, migration and invasion capability of cells in a dose-dependent manner. It is worth mentioning, the trypan blue exclusion assay is a very common assay for evaluating the cell viability $(26,27)$, the blue stained cell indicates incomplete cell membrane, whereas live and apoptosis cells possess intact cell membranes that remain unstained. In this study, proportions of unstained and stained cells among control group and all the ATRA treatment groups were not significantly different (data not shown). Thus, we suggested ATRA at the concentration range of 0.1-10.0 $\mu \mathrm{mol} / \mathrm{l}$ had almost no cytotoxicity to hepa1-6 cells. In addition, the cell density of each group in the wound-healing assay exhibited no difference after 2 days of ATRA treatment, indicating the reduced cell migration was mainly attributed to the decreased migratory capability of hepa1-6 cells, rather than the decreased cell number caused by apoptosis. Furthermore, it was reported that ATRA at low concentration $(0.1 \mu \mathrm{mol} / \mathrm{l})$ could generate these influences. In addition, with the increasing of concentration, the inhibitory effects were enhanced. It has been reported that ATRA at $10 \mu \mathrm{g} / \mathrm{ml}(=33.3 \mu \mathrm{mol} / \mathrm{l})$ inhibited lung adenocarcinoma A549 cell activity (28), $50.0 \mu \mathrm{mol} / 1$ ATRA treatment could shorten the G2/M phase of A549 cell cycle and keep it at G0/G1 phase (29). ATRA ( $1 \mu \mathrm{mol} / \mathrm{l})$ was able to re-differentiate trMCF (transformed breast epithelial cells) at their early stages by regulating breast cancer associated genes. While, the invasive and tumorigenic breast cells did not show any changes in morphology after ATRA treatment (24). Thus, hepatocarcinoma hepa1-6 cells may be more sensitive to the low concentration of ATRA, supporting a beneficial clinical application of ATRA on the therapy of HCC. Next, we further evaluated the effects of ATRA on cell differentiation. Studies have reported ATRA induced maturation of certain kinds of cancer cells. For example, ATRA facilitates the differentiation of acute promyelocytic leukemia cells toward mature granulocytes (30). Oral administration of ATRA induces differentiation of promyelocytic leukemic cells to mature neutrophils (31). Our results also proved that ATRA could effectively induce the expression of late hepatic markers of hepa1-6 cells and improved their hepatic function of metabolism and synthesis. Moreover, this regulation appeared to be strengthened with the increasing concentration of ATRA. Migration and invasion capability are indicators of mesenchymal phenotype, while matured differentiation of hepa1- 6 cells represents the enhancement of their epithelial phenotype. Therefore, our results indicated that ATRA might reverse EMT of hepa1-6 cells.

The downregulation of epithelial markers and the upregulation of mesenchymal markers are both characteristic of EMT.E-cadherin is a key protein in cell polarity and epithelial organization. The reduction or loss of E-cadherin has become one of the hallmarks of EMT, and was frequently associated with dedifferentiation, metastasis and invasion in a variety of human malignancies, including HCC. N-cadherin, vimentin, snail, twist, and fibronectin are known as mesenchymal markers, which are closely linked to several human malignancies (32-34). Moreover, combined detection of these EMT markers has more significance for the mechanism of cancers. Ye et al (34) reported that the low expression of E-cadherin and high expression of $\mathrm{N}$-cadherin were significantly related with local infiltration depth, tumor staging, vascular invasion, and tumor differentiation level. The combined detection of E-cadherin and vimentin has a prognostic value for the patients with oral squamous cell carcinoma (35). Some anticancer drugs targeting these EMT markers are currently used in clinic (36-38), such as silibinin, which inhibits HCC cell 

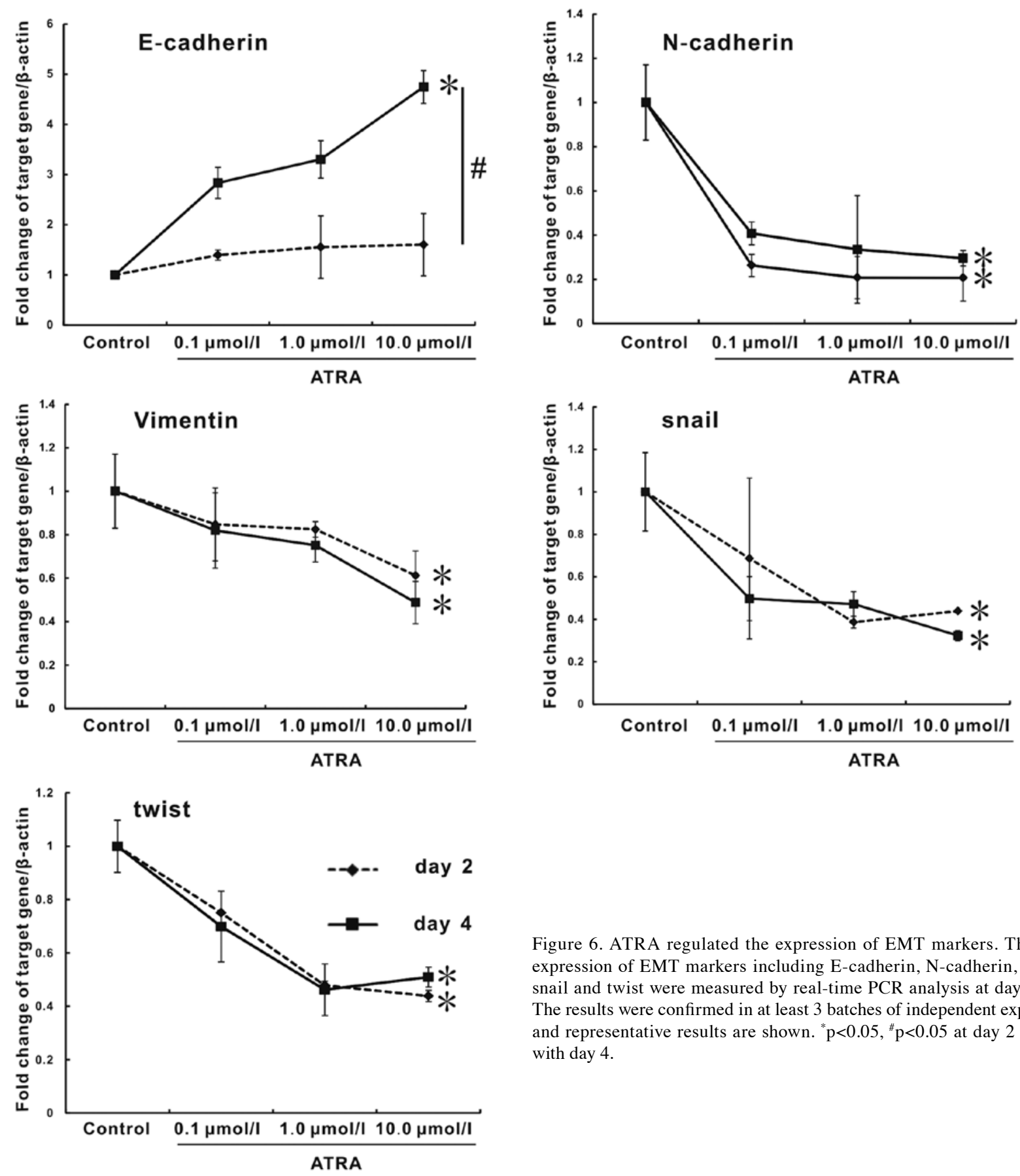

Figure 6. ATRA regulated the expression of EMT markers. The mRNA expression of EMT markers including E-cadherin, N-cadherin, vimentin, snail and twist were measured by real-time PCR analysis at days 2 and 4 . The results were confirmed in at least 3 batches of independent experiments, and representative results are shown. ${ }^{*} \mathrm{p}<0.05,{ }^{\sharp} \mathrm{p}<0.05$ at day 2 compared with day 4 .

proliferation, migration, and invasion via the inhibition of vimentin expression (36). Here we investigate whether ATRA regulates the EMT process and related markers of hepa1-6 cells. We found that ATRA treatment significantly modulated these markers including enhanced expression of E-cadherin and suppressed the expression of $\mathrm{N}$-cadherin, vimentin, snail and twist in HCC cells. In addition, CK18 is one of the epithelial markers associated with EMT and closely related to various cancers $(39,40)$. The expression of CK18 was upregulated by ATRA and was dose-dependent, as was E-cadherin. However, the expression of fibronectin could not be regulated by ATRA (data not shown). Interestingly, the expression of E-cadherin showed significant difference between days 2 and 4 , but no difference of all mesenchymal marker genes was obtained between days 2 and 4, which suggested that the regulation of ATRA on mesenchymal phenotype was triggered probably earlier than the epithelial phenotype. These results indicated that ATRA could inhibit EMT and promote the reverse process in HCC cells. In conclusion, this study demonstrated that ATRA remarkably suppressed hepatocarcinoma proliferation, migration and invasion, as well as effectively induced hepatic tumor cells to restore mature function in vitro through reversing EMT. Interestingly, these effects of ATRA displayed a strengthened trend with the increasing concentration. Our results will contribute to a better understanding of the regulatory mechanisms of ATRA in HCC and provide a strong foundation for the clinical application of ATRA for HCC therapy. 


\section{Acknowledgements}

This study was supported by grants from the National Natural Science Foundation of China (no. 81100309 to Y.B).

\section{References}

1. Forner A, Llovet JM and Bruix J: Hepatocellular carcinoma. Lancet 379: 1245-1255, 2012

2. Llovet JM and Bruix J: Molecular targeted therapies in hepatocellular carcinoma. Hepatology 48: 1312-1327, 2008.

3. Bogachek MV, De Andrade JP and Weigel RJ: Regulation of epithelial-mesenchymal transition through SUMOylation of transcription factors. Cancer Res 75: 11-15, 2015.

4. Thiery JP, Acloque H, Huang RY and Nieto MA: Epithelialmesenchymal transitions in development and disease. Cell 139: 871-890, 2009.

5. Tsai JH and Yang J: Epithelial-mesenchymal plasticity in carcinoma metastasis. Genes Dev 27: 2192-2206, 2013.

6. Trimboli AJ, Fukino K, de Bruin A, Wei G, Shen L, Tanner SM, Creasap N, Rosol TJ, Robinson ML, Eng C, et al: Direct evidence for epithelial-mesenchymal transitions in breast cancer. Cancer Res 68: 937-945, 2008.

7. Ross SA, McCaffery PJ, Drager UC and De Luca LM: Retinoids in embryonal development. Physiol Rev 80: 1021-1054, 2000

8. Feldman DR, Patil S, Trinos MJ, Carousso M, Ginsberg MS, Sheinfeld J, Bajorin DF, Bosl GJ and Motzer RJ: Progression-free and overall survival in patients with relapsed/refractory germ cell tumors treated with single-agent chemotherapy: Endpoints for clinical trial design. Cancer 118: 981-986, 2012.

9. Li M, Sun Y, Guan X, Shu X and Li C: Advanced progress on the relationship between RA and its receptors and malignant tumors. Crit Rev Oncol Hematol 91: 271-282, 2014.

10. Woo YJ and Jang KL: All-trans retinoic acid activates E-cadherin expression via promoter hypomethylation in the human colon carcinoma HCT116 cells. Biochem Biophys Res Commun 425 944-949, 2012.

11. Zanetti A, Affatato R, Centritto F, Fratelli M, Kurosaki M, Barzago MM, Bolis M, Terao M, Garattini E and Paroni G: All-trans-retinoic acid modulates the plasticity and inhibits the motility of breast cancer cells: Role of NOTCH1 and transforming growth factor (TGF $\beta$ ). J Biol Chem 290: 17690-17709, 2015.

12. Chansri N, Kawakami S, Yamashita F and Hashida M: Inhibition of liver metastasis by all-trans retinoic acid incorporated into $\mathrm{O} / \mathrm{W}$ emulsions in mice. Int J Pharm 321: 42-49, 2006.

13. Zhang Y, Guan DX, Shi J, Gao H, Li JJ, Zhao JS, Qiu L, Liu J, Li N, Guo WX, et al: All-trans retinoic acid potentiates the chemotherapeutic effect of cisplatin by inducing differentiation of tumor initiating cells in liver cancer. J Hepatol 59: 1255-1263, 2013.

14. Rodriguez LG, Wu X and Guan JL: Wound-healing assay. Methods Mol Biol 294: 23-29, 2005.

15. Bi Y, Huang J, He Y, Zhu GH, Su Y, He BC, Luo J, Wang Y, Kang Q, Luo Q, et al: Wnt antagonist SFRP3 inhibits the differentiation of mouse hepatic progenitor cells. J Cell Biochem 108 295-303, 2009.

16. Yang MH, Chen CL, Chau GY, Chiou SH, Su CW, Chou TY, Peng WL and Wu JC: Comprehensive analysis of the independent effect of twist and snail in promoting metastasis of hepatocellular carcinoma. Hepatology 50: 1464-1474, 2009.

17. Mima K, Hayashi H, Kuroki H, Nakagawa S, Okabe H, Chikamoto A, Watanabe M, Beppu T and Baba H: Epithelialmesenchymal transition expression profiles as a prognostic factor for disease-free survival in hepatocellular carcinoma: Clinical significance of transforming growth factor- $\beta$ signaling. Oncol Lett 5: 149-154, 2013.

18. Yamada T, Yoshikawa M, Kanda S, Kato Y, Nakajima Y, Ishizaka $S$ and Tsunoda $Y$ : In vitro differentiation of embryonic stem cells into hepatocyte-like cells identified by cellular uptake of indocyanine green. Stem Cells 20: 146-154, 2002.

19. Kamo N, Yasuchika K, Fujii H, Hoppo T, Machimoto T, Ishii T, Fujita N, Tsuruo T, Yamashita JK, Kubo H, et al: Two populations of Thyl-positive mesenchymal cells regulate in vitro maturation of hepatic progenitor cells. Am J Physiol Gastrointest Liver Physiol 292: G526-G534, 2007.

20. Zeisberg M and Neilson EG: Biomarkers for epithelial-mesenchymal transitions. J Clin Invest 119: 1429-1437, 2009.
21. Mongan NP and Gudas LJ: Diverse actions of retinoid receptors in cancer prevention and treatment. Differentiation 75: 853-870, 2007.

22. Connolly RM, Nguyen NK and Sukumar S: Molecular pathways: Current role and future directions of the retinoic acid pathway in cancer prevention and treatment. Clin Cancer Res 19: 1651-1659, 2013.

23. Lo-Coco F, Ammatuna E, Montesinos P and Sanz MA: Acute promyelocytic leukemia: Recent advances in diagnosis and management. Semin Oncol 35: 401-409, 2008.

24. Arisi MF, Starker RA, Addya S, Huang Y and Fernandez SV: All trans-retinoic acid (ATRA) induces re-differentiation of early transformed breast epithelial cells. Int J Oncol 44: 1831-1842, 2014.

25. de Almeida Vasconcelos Fonseca EM, Chagas CE, Mazzantini RP, Heidor R, Ong TP and Moreno FS: All-trans and 9-cis retinoic acids, retinol and beta-carotene chemopreventive activities during the initial phases of hepatocarcinogenesis involve distinct actions on glutathione S-transferase positive preneoplastic lesions remodeling and DNA damage. Carcinogenesis 26: 1940-1946, 2005.

26. Song K, Li W, Wang H, Wang H, Liu T, Ning R and Wang L: Investigation of coculture of human adipose-derived stem cells and mature adipocytes. Appl Biochem Biotechnol 167: 2381-2387, 2012.

27. Zanatta G, Steffens D, Braghirolli DI, Fernandes RA, Netto CA and Pranke P: Viability of mesenchymal stem cells during electrospinning. Braz J Med Biol Res 45: 125-130, 2012.

28. Fan TT, Cheng Y, Wang YF, Gui SY, ChenFH,Zhou Q and Wang Y: A novel all-trans retinoid acid derivative $\mathrm{N}$-(3-trifluoromethylphenyl)-retinamide inhibits lung adenocarcinoma A549 cell migration through down-regulating expression of myosin light chain kinase. Asian Pac J Cancer Prev 15: 7687-7692, 2014.

29. Zhou RJ, Liao WG, Yang ZZ, Min J and Xiao Y: Effects and mechanisms ofATRA on proliferation, cell cycle of lung carcinoma cellline A549. Acta Academiae Medicinae Militaris Tertiae 14: 1399-1401, 2007.

30. Nitto T and Sawaki K: Molecular mechanisms of the antileukemia activities of retinoid and arsenic. J Pharmacol Sci 126: 179-185, 2014.

31. Okuno M, Kojima S, Matsushima-Nishiwaki R, Tsurumi H, Muto Y, Friedman SL and Moriwaki H: Retinoids in cancer chemoprevention. Curr Cancer Drug Targets 4: 285-298, 2004.

32. Tania M, Khan MA and Fu J: Epithelial to mesenchymal transition inducing transcription factors and metastatic cancer. Tumour Biol 35: 7335-7342, 2014.

33. Liu Z, Jin ZY, Liu CH, Xie F, Lin XS and Huang Q: MicroRNA-21 regulates biological behavior by inducing EMT in human cholangiocarcinoma. Int J Clin Exp Pathol 8: 4684-4694, 2015.

34. Ye Z, Zhou M, Tian B, Wu B and Li J: Expression of IncRNACCAT1, E-cadherin and N-cadherin in colorectal cancer and its clinical significance. Int J Clin Exp Med 8: 3707-3715, 2015.

35. Zhou J, Tao D, Xu Q, Gao Z and Tang D: Expression of E-cadherin and vimentin in oral squamous cell carcinoma. Int J Clin Exp Pathol 8: 3150-3154, 2015.

36. Ting HJ, Deep G, Jain AK, Cimic A, Sirintrapun J, Romero LM, Cramer SD, Agarwal C and Agarwal R: Silibinin prevents prostate cancer cell-mediated differentiation of naive fibroblasts into cancer-associated fibroblast phenotype by targeting TGF beta2. Mol Carcinog 54: 730-741, 2015.

37. Thaiparambil JT, Bender L, Ganesh T, Kline E, Patel P, Liu Y, Tighiouart M, Vertino PM, Harvey RD, Garcia A, et al: Withaferin A inhibits breast cancer invasion and metastasis at sub-cytotoxic doses by inducing vimentin disassembly and serine 56 phosphorylation. Int J Cancer 129: 2744-2755, 2011

38. Lee J, Hahm ER, Marcus AI and Singh SV: Withaferin A inhibits experimental epithelial-mesenchymal transition in MCF-10A cells and suppresses vimentin protein level in vivo in breast tumors. Mol Carcinog 54: 417-429, 2015.

39. Tian Q, Xue Y, Zheng W, Sun R, Ji W, Wang X and An R Overexpression of hypoxia-inducible factor $1 \alpha$ induces migration and invasion through Notch signaling. Int J Oncol 47: 728-738, 2015.

40. Wang YP, Yu GR, Lee MJ, Lee SY, Chu IS, Leem SH and Kim DG: Lipocalin-2 negatively modulates the epithelial-tomesenchymal transition in hepatocellular carcinoma through the epidermal growth factor (TGF-beta1)/Lcn2/Twist1 pathway. Hepatology 58: 1349-1361, 2013. 